\title{
Experimental Realization of Full Control of Reflected Waves with Subwavelength Acoustic Metasurfaces
}

\author{
Yong Li ${ }^{1,}{ }^{*}$ Xue Jiang, ${ }^{1}$ Rui-qi Li, ${ }^{1}$ Bin Liang, ${ }^{1,2, \dagger}$ Xin-ye Zou, ${ }^{1}$ Lei-lei Yin, ${ }^{2}$ and Jian-chun Cheng ${ }^{1, *}$ \\ ${ }^{1}$ Key Laboratory of Modern Acoustics, MOE, Department of Physics, \\ Collaborative Innovation Center of Advanced Microstructures, Nanjing University, \\ Nanjing 210093, People's Republic of China \\ ${ }^{2}$ Imaging Technology Group, Beckman Institute, University of Illinois at Urbana-Champaign, \\ Urbana, Illinois 61801, USA
}

(Received 12 August 2014; revised manuscript received 8 October 2014; published 3 December 2014)

\begin{abstract}
Metasurfaces with subwavelength thicknesses have exhibited unconventional phenomena in ways that could not be mimicked by traditional materials. Here we report on the analytical design and experimental realizations of acoustic metasurfaces with hitherto inaccessible functionality of manipulating the reflected waves arbitrarily. By suitably designing the phase-shift profile with a $2 \pi$ span induced by labyrinthine units, the metasurface can reflect acoustic waves in an unusual yet controllable manner. Anomalous reflection and ultrathin planar lenses are both demonstrated with carefully designed metasurfaces. Remarkably, the free manipulation of phase shifts offers great flexibility in the design of nonparaxial acoustic self-accelerating beams with arbitrary trajectories. With extraordinary wave-steering ability, the metasurface should open exciting possibilities for designing compact acoustic components with versatile potential and may find a variety of applications ranging from ultrasound imaging to caustic engineering where designing the shape of a focused trajectory of sound is required.
\end{abstract}

DOI: 10.1103/PhysRevApplied.2.064002

\section{INTRODUCTION}

Over the past few years, there has been a rapid expansion of the field of metasurfaces, which are optically thin metal gratings with subwavelength periodicity that exhibit strong influence on incident light to generate diverse functionalities such as bending light abnormally, manipulating polarization, and creating ultrathin planar lenses [1-7]. Optical metasurfaces with unusual properties that cannot be duplicated by using traditional optical materials or devices have shown great promise in fully manipulating electromagnetic waves. An acoustic wave is another important form of a classical wave, and molding the flow of reflected acoustic waves is of paramount significance to the current research efforts in a large variety of applications ranging from room acoustics to medical ultrasound imaging. For the purpose of arbitrarily tailoring acoustic propagation, however, full control of the phase of the acoustic field is required, which is hard to accomplish with the existing acoustic materials and devices due to the limitations in their acoustic properties [8]. In this context, it is expected that an acoustic metasurface with the ability to control the reflected wave at will, if

\footnotetext{
*Present address: Department of Physics, Hong Kong University of Science and Technology, Clear Water Bay, Kowloon, Hong Kong, China.

${ }^{\dagger}$ Corresponding author. liangbin@nju.edu.cn

${ }^{\star}$ Corresponding author. jccheng@nju.edu.cn
}

realized, will be highly intriguing and may lead to revolutionary innovations in the acoustic field and, furthermore, acoustic-based applications. The recent emergence of acoustic metamaterials [9-19] with properties unavailable in nature has broadened the horizon of acoustic waves and enabled fascinating phenomena such as subdiffraction imaging [20-22], one-way transportation [23-27], cloaking [28-30], superabsorption [31,32], etc. So far, however, the research on acoustic metasurface is scarce [33,34], and there still remain considerable practical challenges to experimentally realize the full manipulations of acoustic reflected waves with subwavelength metasurfaces.

In this work, we analytically design and experimentally demonstrate acoustic metasurfaces that are capable of arbitrarily manipulating reflected waves in the direction and shape of the wave front. A general scheme of designing an acoustic metasurface is developed on the basis of different labyrinthine units. It is worth stressing that the proposed scheme provides a substantial conceptual advantage over the first theoretical frame presented by us with some seemingly similar labyrinthine units for which the physical mechanism is complicated and cannot be understood easily due to the extrastraight air channels [33]. Furthermore, the proposed scheme has an advantage in device performance because the original design needs many zigzag channels to realize the desired phase shifts, which inevitably leads to difficulties in experiments and remarkable viscosity [9]. Because of our different design idea, the resulting metasurface can reflect acoustic waves in 
an unusual yet controllable manner, for which both the underlying physics and the device performance can be well understood with the analytical method developed here. Despite the vanishing thickness in comparison to the incident wavelength, acoustic metasurfaces are shown both theoretically and experimentally to have the distinct properties of realizing abnormal reflection governed by the generalized Snell's law and ultrathin planar lenses with a desired focal point. Furthermore, we demonstrate the remarkable functionality of generating nonparaxial accelerating beams along an arbitrary trajectory, which reveals great flexibility in the design of acoustic beams enabled by the free manipulation of a propagating phase with a metasurface. Capable of engineering an acoustic wave front with subwavelength thicknesses, the acoustic metasurface should open exciting new possibilities for designing versatile compact acoustic components and may be applied to important fields ranging from ultrasound imaging to field caustic engineering where designing the shape of a focused trajectory of sound is required.
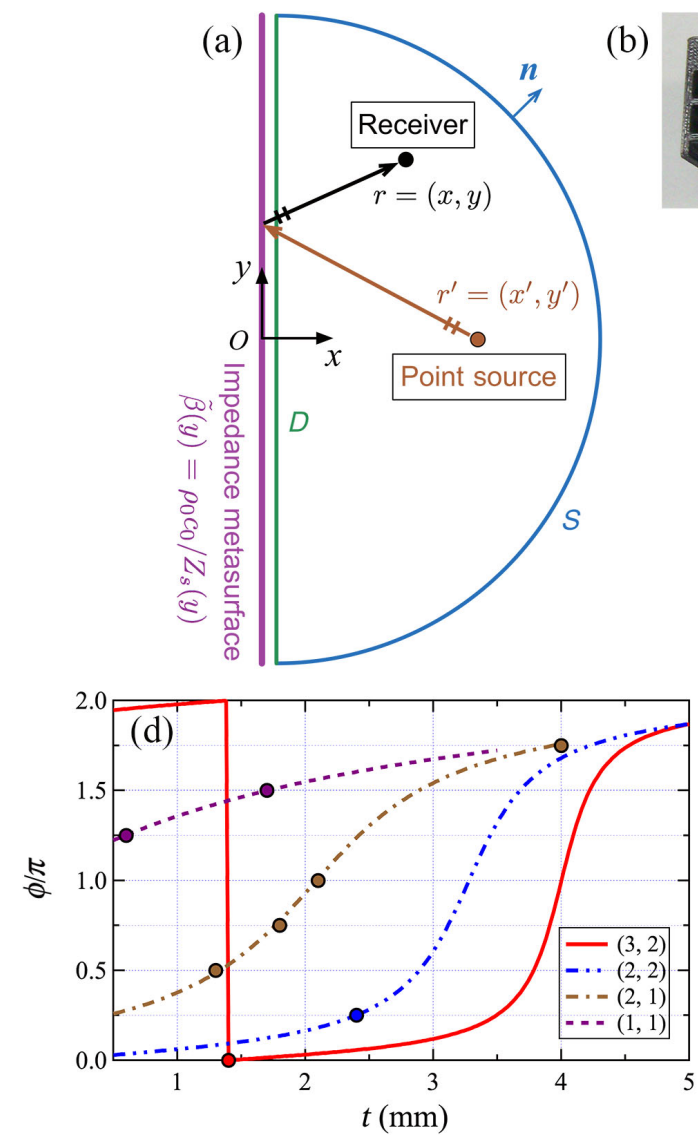

\section{METHOD AND DESIGN}

\section{A. Analytical reflected field of the metasurface}

Figure 1(a) illustrates the presented acoustic metasurface, which can be characterized by an acoustic normalized admittance $\tilde{\beta}(y)=\rho_{0} c_{0} / Z_{s}$. Here, $\rho_{0} c_{0}$ is the acoustic impedance of air with $\rho_{0}$ and $c_{0}$ referring to the density and velocity of air, respectively, and $Z_{s}(y)$ is the acoustic impedance of the metasurface. The admittance considered in our case is a pure acoustic susceptance, which is capable of effectively modulating the phase of the reflected waves. The relationship between the susceptance and the phase shift can be expressed as $\tilde{\beta}(y)=i \tan [\phi(y) / 2]$ with $\phi(y)$ referring to the phase shift provided by the metasurfaces. In the following, the frequency domain is considered, and the harmonic factor $e^{-i \omega t}$ with $i=\sqrt{-1}$ is omitted. Considering the two-dimensional case with a point source located at $\left(x^{\prime}, y^{\prime}\right)$, the Green's function of the free space can be expressed as [8]

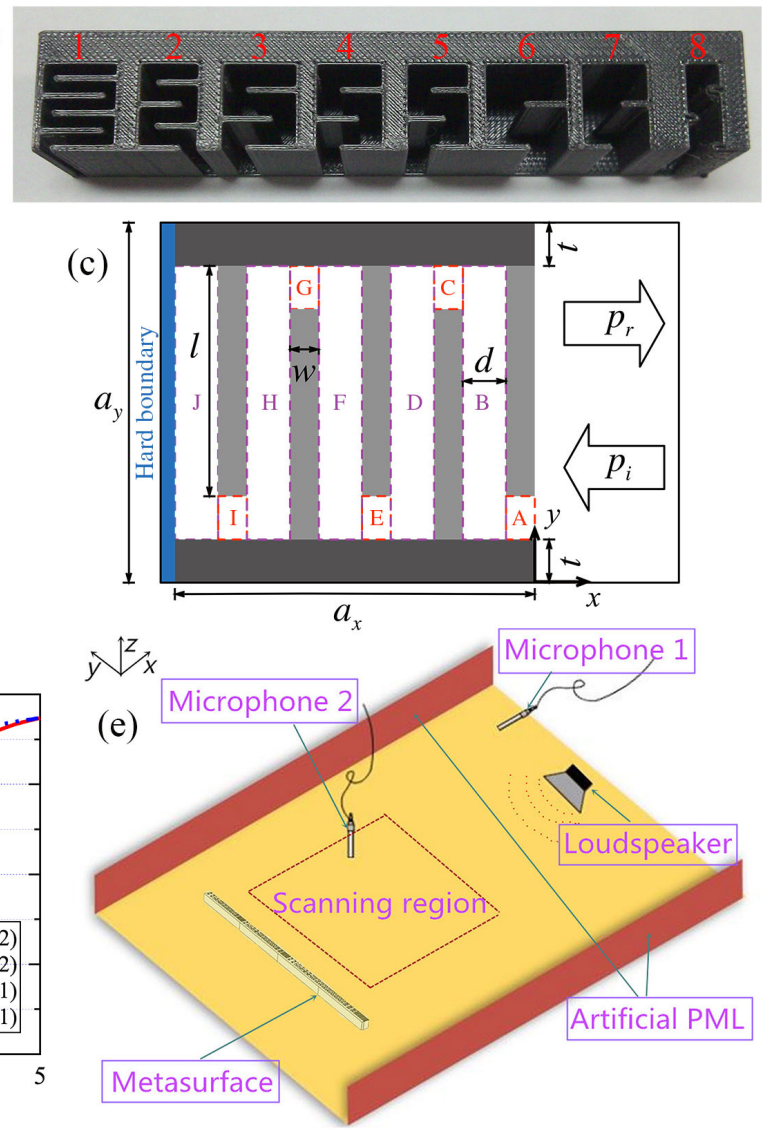

FIG. 1. (a) A map for deriving the reflected pressure field with a point source. The proposed metasurface is characterized by acoustic admittance $\tilde{\beta}(y)$. (b) Photo of the proposed eight labyrinthine units labeled 1 to 8 , which can provide phase shifts ranging from 0 to $2 \pi$ with a step of $\pi / 4$. (c) The details of a specific labyrinthine unit (width $a_{x}$ and height $a_{y}$ ), which are composed of several identical crossarranged plates (width $w$ and length $l$ ) that form a labyrinthine channel with width $d$. Another two plates (thickness $t$ ) are used to seal the channels in the $y$ direction. (d) The phase shifts induced by four different kinds of labyrinthine units as a function of $t$. The $(m, n)$ case reveals that there are $m$ identical plates in the upper boundary and $n$ identical plates in the lower boundary. The eight dots refer to the chosen eight units shown in (b). (e) The experimental setup for scanning the pressure fields. 


$$
G\left(x, y ; x^{\prime}, y^{\prime}\right)=\frac{i}{4} H_{0}^{(1)}\left(k_{0} R\right),
$$

where $R$ is the distance between the field point $(x, y)$ and the source point $\left(x^{\prime}, y^{\prime}\right) \quad R \equiv \sqrt{\left(x-x^{\prime}\right)^{2}+\left(y-y^{\prime}\right)^{2}}$, $H_{0}^{(1)}(\cdot)$ is the first kind of Hankel function of zero order, and $k_{0}=\omega / c_{0}$ refers to the wave number in air with $\omega$ being the angular frequency. Assuming a metasurface of length $2 L$ located at $x=0$ and extending from $y=-L$ to $y=L$, the total field can be expressed as [8]

$$
\begin{aligned}
p(x, y)= & \int_{V} G\left(x, y ; x^{\prime}, y^{\prime}\right) \Im\left(x^{\prime}, y^{\prime}\right) d V^{\prime} \\
& +\int_{S+D}\left[G\left(x, y ; x^{\prime}, y^{\prime}\right) \frac{\partial p\left(x^{\prime}, y^{\prime}\right)}{\partial n^{\prime}}\right. \\
& \left.-p\left(x^{\prime}, y^{\prime}\right) \frac{\partial G\left(x, y ; x^{\prime}, y^{\prime}\right)}{\partial n^{\prime}}\right] d S^{\prime},
\end{aligned}
$$

where $\Im(x, y)$ is the bulk distribution of acoustic sources, which can be simulated by a Dirac function $\Im(x, y)=$ $Q \delta\left(x-x^{\prime}\right) \delta\left(y-y^{\prime}\right)$ with a normalized strength $Q=1$, and $\partial / \partial n^{\prime}$ is the normal derivative of the integral paths $S$ and $D$, as shown in Fig. 1(a). By substituting Eq. (1) into Eq. (2), the reflected waves caused by the metasurface can be obtained analytically as follows:

$$
\begin{aligned}
p_{r}(x, y)= & -\left.\frac{k_{0}}{4} \int_{-L}^{L} \tilde{\beta}\left(y^{\prime}\right) p\left(0, y^{\prime}\right) H_{0}^{(1)}\left(k_{0} R\right)\right|_{x^{\prime}=0} d y^{\prime} \\
& +\left.\frac{i}{4} \int_{-L}^{L} p\left(0, y^{\prime}\right) \frac{\partial H_{0}^{(1)}\left(k_{0} R\right)}{\partial x^{\prime}}\right|_{x^{\prime}=0} d y^{\prime},
\end{aligned}
$$

where $p(0, y)$ is the pressure profile at the metasurface boundary, which can be expressed as

$$
\begin{aligned}
\frac{p(0, y)}{2}= & \left.\frac{i}{4} H_{0}^{(1)}\left(k_{0} R\right)\right|_{x=0} \\
& -\left.\frac{k_{0}}{4} \int_{-L}^{L} \beta\left(y^{\prime}\right) p\left(0, y^{\prime}\right) H_{0}^{(1)}\left(k_{0} R\right)\right|_{x^{\prime}=0} d y^{\prime} \\
& +\left.\frac{i}{4} \int_{-L}^{L} p\left(0, y^{\prime}\right) \frac{\partial H_{0}^{(1)}\left(k_{0} R\right)}{\partial x^{\prime}}\right|_{x^{\prime}=0} d y^{\prime} .
\end{aligned}
$$

Equation (4) is a Fredholm integral equation of the second kind and can be solved through discretization. From Eq. (2), it is found that reflected waves caused by the metasurface can be tailored efficiently by appropriately choosing the expression of $\tilde{\beta}(y)$ or $\phi(y)$. Remarkably, the reflected field can be arbitrarily designed exactly as desired, due to the fact that no assumptions such as the far-field approximation or Born approximation have been employed in the derivation process. It is also noteworthy that the metasurface with a length of $2 L$ is employed in the derivation, and the effect of the finite length has already been taken into consideration.

\section{B. Reflection coefficient of a labyrinthine unit}

As shown in Fig. 1(b), the designed metasurface consists of eight different labyrinthine units, in which several stiff plates are arranged to form a zigzag channel $[16,17,19,35]$, which will be demonstrated in what follows, to be sufficient for constructing the reflected field at will. For acoustic metasurfaces, the phase of the reflected waves by each unit is crucial for the acoustic phenomenon. It is, therefore, essential to develop a method to obtain the reflected phase to benefit the design of the metasurfaces. Here, we develop a method to analytically solve the reflected waves by a labyrinthine unit. A specific labyrinthine unit is considered [Fig. 1(c)], which is divided into two kinds of short pipes: the first are labeled, in turn, as A, C, E, G, and I, and the second are labeled, in turn, as B, D, F, H, and J, respectively. The alternate arrangement of these two kinds of pipes can be equivalently regarded as an abrupt variation in the cross section of the pipes. Let $\left(p_{L}, u_{L}\right)$ denote the sound pressure and the axial components of the velocities, respectively. Here, the subscripts $L=i$ and $r$ refer to the incident and the reflected fields, respectively, and $L=A-J$ indicates the fields inside the corresponding channels. The velocity components $u$ are associated with the corresponding sound pressure $p$ via the relationship $i k_{0} \rho_{0} c_{0} u=\partial p / \partial x$.

In acoustics, waves can propagate within a subwavelength waveguide in the absence of a cutoff frequency. Since the length in the $y$ direction is 2 orders of magnitude smaller than the wavelength, the field $\left(p_{L}, u_{L}\right)$ in the regions $\mathrm{A}$ (viz., $-w \leq x \leq 0$ and $t \leq y \leq t+d$ ), $\mathrm{B}$ (viz., $-w-d \leq x \leq w$ and $t \leq y \leq t+d+l$ ), and $\mathrm{C}$ (viz., $-2 w-d \leq x \leq-w-d$ and $t+l \leq y \leq t+l+d$ ) can be expanded with normal modes and expressed as [8]

$$
\begin{gathered}
p_{A}(x, y)=A^{+} e^{i k_{0}(x+w)}+A^{-} e^{-i k_{0} x} \\
u_{A}(x, y)=\frac{1}{\rho_{0} c_{0}}\left[A^{+} e^{i k_{0}(x+w)}-A^{-} e^{-i k_{0} x}\right] \\
p_{B}(x, y)=\sum_{n=0}^{\infty} \phi_{n}(y)\left[B_{n}^{+} e^{i k_{x n}(x+w+d)}+B_{n}^{-} e^{-i k_{x n}(x+w)}\right] \\
u_{B}(x, y)=\sum_{n=0}^{\infty} \phi_{n}(y) \frac{k_{x n}}{k_{0} \rho_{0} c_{0}}\left[B_{n}^{+} e^{i k_{x n}(x+w+d)}-B_{n}^{-} e^{-i k_{x n}(x+w)}\right] \\
p_{C}(x, y)=C^{+} e^{i k_{0}(x+2 w+d)}+C^{-} e^{-i k_{0}(x+w+d)} \\
u_{C}(x, y)=\frac{1}{\rho_{0} c_{0}}\left[C^{+} e^{i k_{0}(x+2 w+d)}-C^{-} e^{-i k_{0}(x+w+d)}\right]
\end{gathered}
$$

where $\phi_{n}(y)$ is the transverse eigenmode

$$
\phi_{n}(y)=\sqrt{2-\delta_{0 n}} \cos \left[k_{y n}(y-t)\right] .
$$


Here, the wave number along the $y$ direction can be expressed as $k_{y n}=n \pi /(l+d) \quad(n=0,1,2, \ldots$,$) and$ $k_{x n}=\sqrt{k_{0}^{2}-k_{y n}^{2}}$. The symbols $A^{+}, A^{-}, C^{+}$, and $C^{-}$denote the coefficients in the thin channel, and $B_{n}^{+}$and $B_{n}^{-}$denote the coefficients of the $n$th mode with the normal wave number $k_{n}$. Note that the geometry size along the $y$ direction of regions represented as $\mathrm{A}$ and $\mathrm{C}$ (viz., $d$ ) is much smaller compared to the working wavelength. It is, therefore, possible in this case to simplify the analytical derivation by considering the plane-wave component only; i.e., the summations over $n$ have not applied in Eqs. (5), (6), (9), and (10). To obtain more accurate results for the regions represented as B whose geometrical size (viz., $l+d$ ) is comparable to $a_{y}$, the summation of normal modes is employed.

Considering that the continuity of pressure should be satisfied on the boundary between region $\mathrm{A}$ and region $\mathrm{B}$ at $x=-w$ and the boundary between regions $\mathrm{B}$ and $\mathrm{C}$ at $x=-w-d$, a transfer matrix can be constructed to connect the coefficients between regions $\mathrm{A}$ and $\mathrm{C}$ as follows:

$$
\left[\begin{array}{l}
C^{+} \\
C^{-}
\end{array}\right]=M_{1}\left[\begin{array}{l}
A^{+} \\
A^{-}
\end{array}\right]
$$

where

$$
M_{1}=\left[\begin{array}{cc}
\frac{\beta^{2}-\alpha^{2}+1}{2 \beta} & -\frac{\beta^{2}-(\alpha+1)^{2}}{2 \beta} e^{-i k_{0} w} \\
\frac{\beta^{2}-(\alpha-1)^{2}}{2 \beta} e^{i k_{0} w} & -\frac{\beta^{2}-\alpha^{2}+1}{2 \beta}
\end{array}\right],
$$

where

$$
\begin{aligned}
& \alpha=\sum_{n=0}^{\infty} \frac{k_{0} d}{k_{x n}(l+d)} \frac{1+e^{i 2 k_{x n} d}}{1-e^{i 2 k_{x n} d}} \Phi_{n}^{1} \Phi_{n}^{1}, \\
& \beta=\sum_{n=0}^{\infty} \frac{k_{0} d}{k_{x n}(l+d)} \frac{2 e^{i k_{x n} d}}{1-e^{i 2 k_{x n} d}} \Phi_{n}^{1} \Phi_{n}^{2},
\end{aligned}
$$

with

$$
\Phi_{n}^{1}=\frac{1}{d} \int_{t}^{t+d} \phi_{n}(y) d y, \quad \Phi_{n}^{2}=\frac{1}{d} \int_{t+l}^{t+l+d} \phi_{n}(y) d y .
$$

It can be found that the transfer matrix from regions $\mathrm{C}$ to $\mathrm{E}$ is the same as the above matrix. Therefore, the coefficients in region I can be expressed as

$$
\left[\begin{array}{l}
I^{+} \\
I^{-}
\end{array}\right]=M_{1}^{4}\left[\begin{array}{l}
A^{+} \\
A^{-}
\end{array}\right]
$$

Considering the continuity of pressure and volume velocity at the interface between regions I and $\mathrm{J}$, and the fact that the left boundary of region J is a hard boundary, the coefficients in region I can be expressed as

$$
\frac{I^{+}}{I^{-}}=-\frac{1+\alpha}{1-\alpha} e^{i k_{0} w}
$$

The pressure field in the incident region (viz., $x \geq 0$ and $0 \leq y \leq a_{y}$ ) can be expressed as

$$
p(x, y)=p_{i}+p_{r}=e^{-i k_{0} x}+\sum_{n=0}^{\infty} R_{n} \psi_{n}(y) e^{i k_{x n}^{\prime} x},
$$

where $\psi_{n}(r)$ is the transverse eigenmode

$$
\psi_{n}(y)=\sqrt{2-\delta_{0 n}} \cos \left(k_{y n}^{\prime} y\right),
$$

with the wave number along the $y$ direction given as $k_{y n}^{\prime}=$ $n \pi /(l+d+2 t)$ and $k_{x n}^{\prime}=\sqrt{k^{2}-k_{y n}^{\prime 2}}$. Following the same procedure, the transfer matrix connecting the incident region and region $\mathrm{A}$ is

$$
\left[\begin{array}{c}
A^{+} \\
A^{-}
\end{array}\right]=M_{2}\left[\begin{array}{c}
1 \\
R_{0}
\end{array}\right]
$$

with

$$
M_{2}=\left[\begin{array}{cc}
\left(1-\frac{\beta^{\prime}}{2}\right) e^{-i k_{0} w} & \frac{\beta^{\prime}}{2} e^{-i k_{0} w} \\
1+\frac{\alpha^{\prime}}{2} & -\frac{\alpha^{\prime}}{2}
\end{array}\right],
$$

with

$$
\alpha^{\prime}=\frac{a_{y}}{d}-\sum_{n=0}^{\infty} \frac{k_{0}}{k_{x n}^{\prime}}\left(\Psi_{n}^{\prime}\right)^{2}, \quad \beta^{\prime}=\frac{a_{y}}{d}+\sum_{n=0}^{\infty} \frac{k_{0}}{k_{x n}^{\prime}}\left(\Psi_{n}^{\prime}\right)^{2} .
$$

Combining Eqs. (16), (17), and (20), the total transfer matrix can be expressed as

$$
R_{0}=-\frac{(1-\alpha) m_{11}+(1+\alpha) e^{i k_{0} w} m_{21}}{(1-\alpha) m_{12}+(1+\alpha) e^{i k_{0} w} m_{22}},
$$

with

$$
M=M_{1}^{4} M_{2}=\left[\begin{array}{ll}
m_{11} & m_{12} \\
m_{21} & m_{22}
\end{array}\right] .
$$

\section{Realization of the full control of reflected waves}

Once the complex reflection coefficient is obtained, the impedance of the presented metasurface can be expressed as

$$
Z_{s}=\frac{1-R_{0}}{1+R_{0}}
$$

Then the admittance of each labyrinthine unit can be expressed as $\tilde{\beta}=\rho_{0} c_{0} / Z_{s}=i \tan (\phi / 2)$ with $\phi=\arg \left(R_{0}\right)$ being the phase shifts induced by each labyrinthine unit. It 
will be demonstrated later that the desired phase shift covering the full $2 \pi$ range can be achieved by adjusting a single parameter of $t$, which guarantees a totally planar metasurface. The parameters of $a_{x}$ and $a_{y}$ are fixed to be $\lambda_{0} / 8$ with $\lambda_{0}=0.1 \mathrm{~m}$ to ensure the subwavelength feature of the resulting device. The width of these identical plates $w$ [gray regions in Fig. 1(c)] is chosen as $1 \mathrm{~mm}$ for facilitating the sample fabrication. Figure 1(d) illustrates the phase change as a function of $t$ for four kinds of labyrinthine units. Actually, by appropriately choosing the value of $t$, the case $(m, n)=(3,2)$ is sufficient for providing a phase change ranging from 0 to $2 \pi$ with a step of $\pi / 4$. In the experimental demonstrations, labyrinthine units with less zigzagged structures are employed for facilitating the fabrication of samples as well as minimizing the viscosityloss effect. According to the phase shift shown in Fig. 1(d), eight units can be chosen covering a $2 \pi$ range with a step of $\pi / 4$, and the values of $t$ for each unit are labeled with eight dots. Then, the width of the labyrinthine channel can be expressed as $d=a_{x} /(m+n)-w$, and the length of the plates is $l=a_{y}-2 t-d$ for these eight types of units. Note that by employing common anisotropic metamaterials, it is still possible to realize similar results of wave-front manipulation approximately. In such cases, however, the relatively low refractive indexes significantly limit the amount of phase shift that can be provided within a certain distance $[34,36]$. As a result, the total thickness of the whole system based on these materials must be considerably large for realizing the desired phase shift. The labyrinthine units used here take advantage of a high refractive index [16,37-40] and a subsequently small thickness $\left(\lambda_{0} / 8\right)$, which enables the design of an acoustic metasurface.

\section{Sample fabrication and experimental setup}

Figure 1(e) schematically demonstrates the experimental setup. The metasurfaces are fabricated with thermoplastics via $3 \mathrm{D}$ printing (Stratasys Dimension Elite, $0.08 \mathrm{~mm}$ in precision) to meet the requirement of the theoretical design. The samples are placed between two paralleled plexiglass plates $(1.2 \mathrm{~m} \times 1.5 \mathrm{~m})$, which forms the $2 \mathrm{D}$ experimental environment, and the setup is schematically shown in Fig. 1(e). The distance of the experimental system in the $z$ direction is $2.1 \mathrm{~cm}$, the same as the thicknesses of the metasurfaces so that the sample can come in contact with the two plates close together. Wedge-shaped soundabsorbing foam is installed at the boundary of the plexiglass plates to mimic an anechoic environment. A loudspeaker (30 $\mathrm{mm}$ in diameter) is mounted in a box baffle and placed outside but very near to the upper plate $1 \mathrm{~m}$ away from the metasurfaces. It emits a monochromatic wave as the acoustic source and propagates into the $2 \mathrm{D}$ experimental environment between the paralleled plexiglass plates through a small hole (20 $\mathrm{mm}$ in diameter) in the upper plate. For each measurement, two 1/4-in microphones (Brüel \& Kjær type 4961) are placed at designated positions to sense the local pressure: one is mounted at a fixed position to detect the pressure as signal 1 and the other is moveable to scan the pressure field as signal 2. By using the software PULSE LABSHOP, the cross spectrum of the two signals gathered by the two microphones is obtained, for which signal 1 works as a reference and signal 2 as an input signal. The pressure field is retrieved by analyzing the cross spectrum and recording the magnitude and phase at different spatial positions within the measured region. Then, the reflected pressure field is obtained by subtracting two pressure fields with and without the metasurfaces.

\section{RESULTS AND DISCUSSION}

\section{A. The generalized Snell's law of reflection and anomalous reflection}

The full $2 \pi$-range phase shift offered by the metasurface comprised of a monolayer of metamaterial enables us to revisit Snell's law. Here we will combine theory and experiment to demonstrate that a properly designed metasurface can support anomalous reflection for incident acoustic waves governed by the generalized Snell's law. The schematics for the derivation of the angle of reflection are shown in Fig. 2(a), where $\theta_{r}$ represents the reflection angle. To support the anomalous reflection, the phase gradient along the $y$ direction should satisfy $d \phi(y) / d y=-k_{0} \sin \left(\theta_{r}\right)$. Here, the reflection angle is set to be $\pi / 6$. In this case, since the incident waves are radiated from a loudspeaker located at $\left(x^{\prime}, y^{\prime}\right)=(1 \mathrm{~m}, 0 \mathrm{~m})$, there exists an extra phase change $\phi_{\text {extra }}=-k_{0}\left(\sqrt{y^{2}+1}-1\right)$ that needs to be compensated by the metasurface as well. Therefore, the desired phase profile yielded by the metasurface should be $\phi(y)=-k_{0}(y+0.3) \sin (\pi / 6)-$ $k_{0}\left(\sqrt{y^{2}+1}-1\right)$ as shown in Fig. 2(b). Such a profile can then be yielded by constructing the acoustic metasurface with appropriately designed labyrinthine units. The pressure-field distribution predicted analytically by Eq. (2) is plotted in Fig. 2(c), where the black box indicates the region within which the measurement is performed. The results of the experiments, along with that of the numerical simulations for the metasurface composed by the labyrinthine units, are shown in Figs. 2(d) and 2(c), respectively. Excellent agreement is observed between the numerical and experimental results, with the black arrows referring to the theoretical angle of the reflection. They both demonstrate that by carefully designing the geometric parameters, the acoustic metasurface can bend the incident wave to the desired angle, following the generalized Snell's law with an additional parallel wave vector provided by the phase gradient of the metasurface and, furthermore, can even be used to realize the conversion from propagating waves to surface waves $[1,4]$. The generalization of the law of reflection by acoustic metasurfaces is applicable to a large 

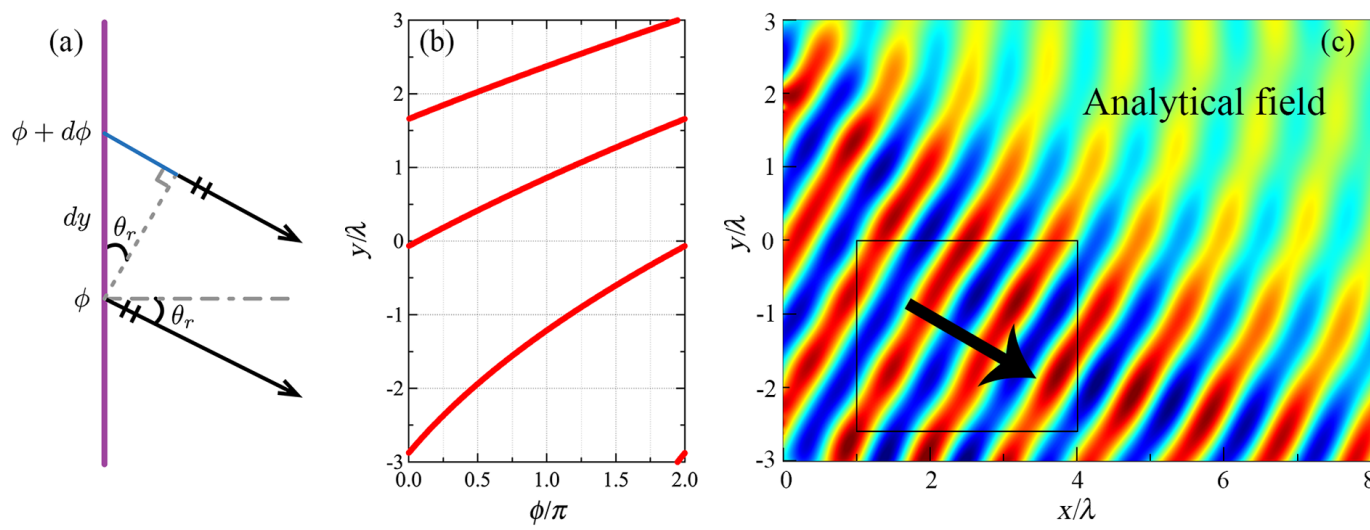

(c)
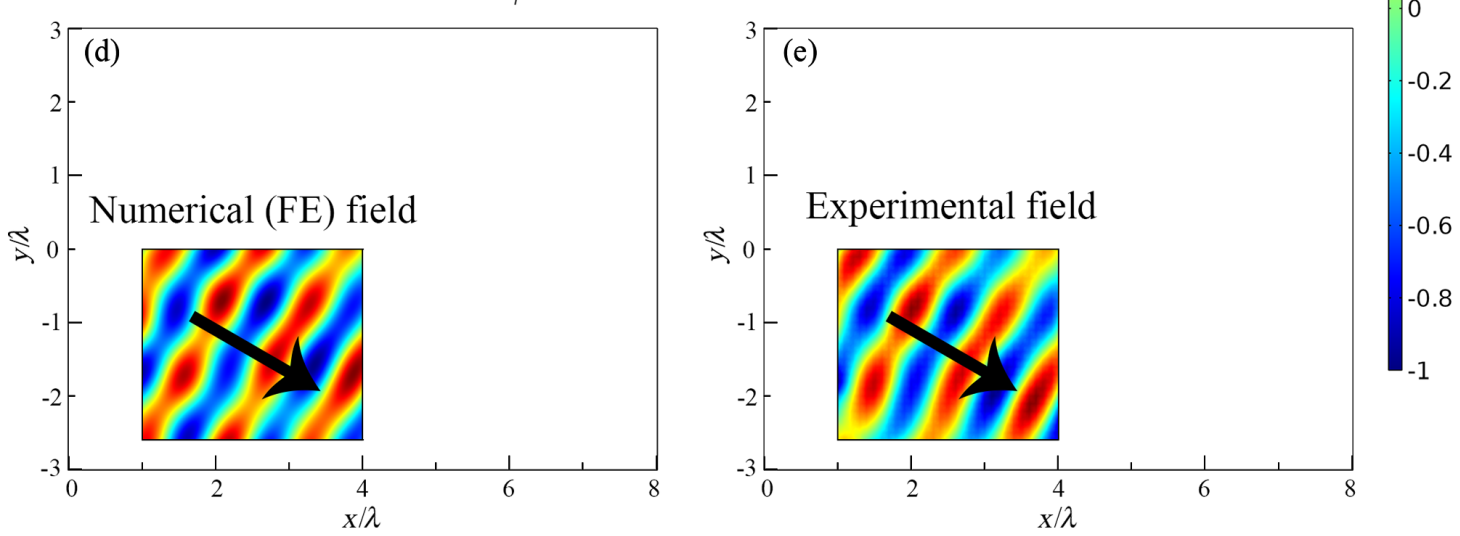

FIG. 2. (a) The schematics for the derivation of the angle of reflection. The length of the blue line refers to the difference of the acoustic path distance provided by the metasurface in the $y$ direction. (b) The desired continuous phase profile that needs to be yielded by the metasurfaces with length $2 L=6 \lambda$. (c) The pressure-field distribution predicted by Green's-function theory. The black box indicates the region within which the measurement is performed. (d) The numerical simulation of the metasurface composed by labyrinthine units, and (e) the experimental results of the pressure-field distribution. Black arrows in (c)-(e) refer to the theoretical angle of the reflection.

variety of interfaces with subwavelength engineered structures and offers a novel opportunity to control over acoustic waves versatilely.

\section{B. Ultrathin planar acoustic lens}

Figure 3(a) illustrates the design of a metasurface-based lens. To obtain a focal spot in the axis located at $(x, y)=$ $\left(f_{x}, 0\right)=(0.3 \mathrm{~m}, 0 \mathrm{~m})$, the equiphase surface should be the gray line indicating that the phase profile should be expressed as $\phi(y)=-k_{0}\left(\sqrt{y^{2}+f_{x}^{2}}-f_{x}\right)$, and the phase profile provided by the metasurface should be $\phi(y)=$ $-k_{0}\left(\sqrt{y^{2}+0.9}-0.3\right)-k_{0}\left(\sqrt{y^{2}+1}-1\right)$, as shown in Fig. 3(b). The pressure-field distribution predicted analytically by Eq. (2) is shown in Fig. 3(c), showing the effectiveness of such a design. The experimental results, which are in excellent agreement with the numerical simulation, demonstrate that the metasurface can be tailored to mimic an ultrathin planar lens that has a thickness only $\lambda_{0} / 8$ of the incident wavelength yet is capable of effectively steering the convergence of acoustic waves. To quantify the performance of the lens, acoustic intensity distributions along the axis and the transverse cross section through the focal point are measured and shown in Figs. 3(c) and 3(d), which agree almost perfectly with the theoretical and numerical predictions. Furthermore, due to the full control of the phase profile, the metasurface can be conveniently reconfigured to become an acoustic lens with an arbitrary focal point. The corresponding results for a typical example of a decentered lens are illustrated in Fig. 4 with a focal point at $(x, y)=\left(f_{x}, f_{y}\right)=(0.3 \mathrm{~m}, 0.1 \mathrm{~m})$, in which a perfect match between the theoretical and measured results can be observed as well. The capability of focusing lowfrequency sound using a subwavelength-scale metasurface that manipulates the phase of the propagating wave may pave the way to diverse applications such as acoustic imaging and caustic engineering that requires a design of the shape of a focused trajectory of sound. The focusing effect can also be realized by metamaterial-based lenses; however, such lenses may suffer from a limitation in the refractive index, resulting in a considerably large size [36]. In comparison, the high refractive index provided by the proposed acoustic metasurface enables full control over the phase of the wave with a lens thickness as small as $\lambda_{0} / 8$, which offers greater freedom to design the focal point. 

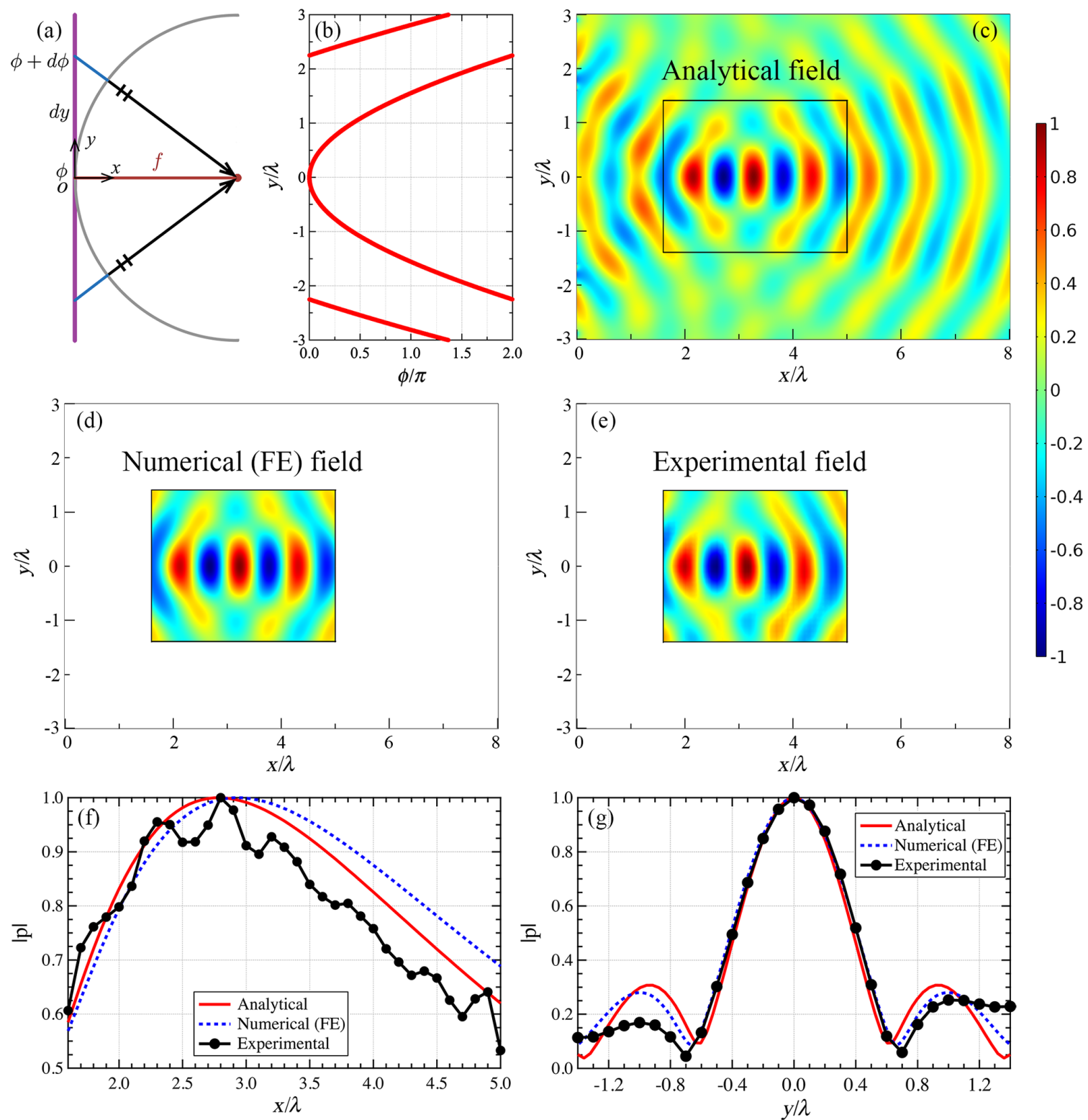

(c)
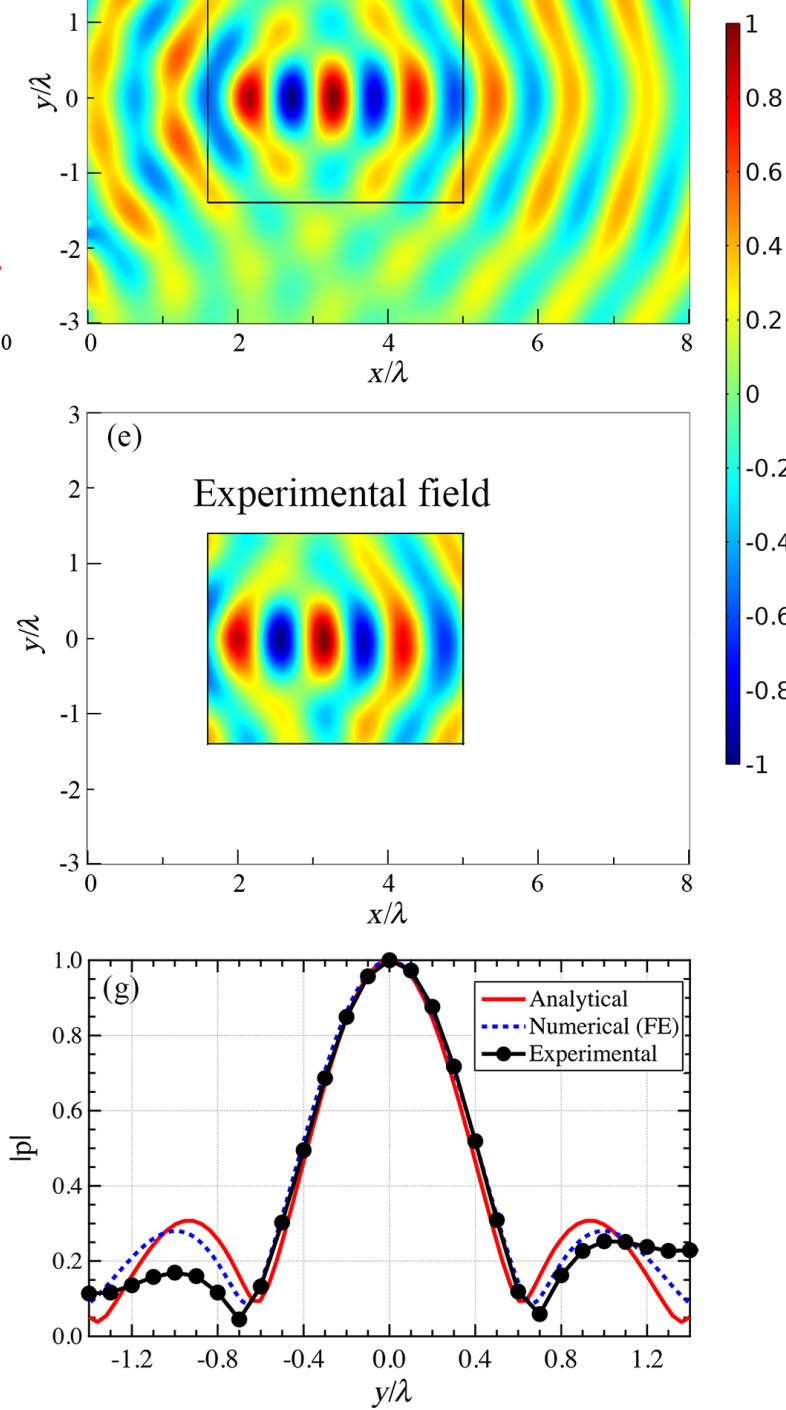

FIG. 3. (a) The schematics for the derivation of the acoustic focusing with focus length $f$. The length of the blue line is the difference in the acoustic path distance provided by the metasurface along the $y$ direction. (b) The desired continuous phase profile that needs to be yielded by the metasurfaces with length $2 L=6 \lambda$. (c) The pressure-field $p(x, y)$ distribution predicted by Green's-function theory. The black box indicates the region within which the measurement is performed. (d) The numerical simulation of metasurface composed by labyrinthine units and (e) the experimental results of the pressure-field distribution. Acoustic pressure amplitude profiles (f) along the acoustic axis and $(\mathrm{g})$ the radial direction in the focal plane.

\section{Self-bending beams with arbitrary trajectory}

Accelerating optical beams, such as Airy beams [41,42], nonparaxial Mathieu and Webber beams [43], etc., have been experimentally demonstrated recently and have significant impact on the optical fields. In order to show the potential of the acoustic metasurface to fully control the acoustic waves, we demonstrate both theoretically and experimentally the generation of a selfaccelerating and bending acoustic beam that propagates along an arbitrary caustic trajectory [44,45]. It is worth emphasizing that the proposed scheme can be used to design metasurfaces with the ability to generate paraxial acoustic beams represented by Airy beams, as well as nonparaxial ones. To facilitate the experimental demonstration, however, here we investigate only the design and fabrication of metasurfaces capable of generating a nonparaxial beam, instead of the case of paraxial beams which are less general but much harder to measure. Figure 5(a) 

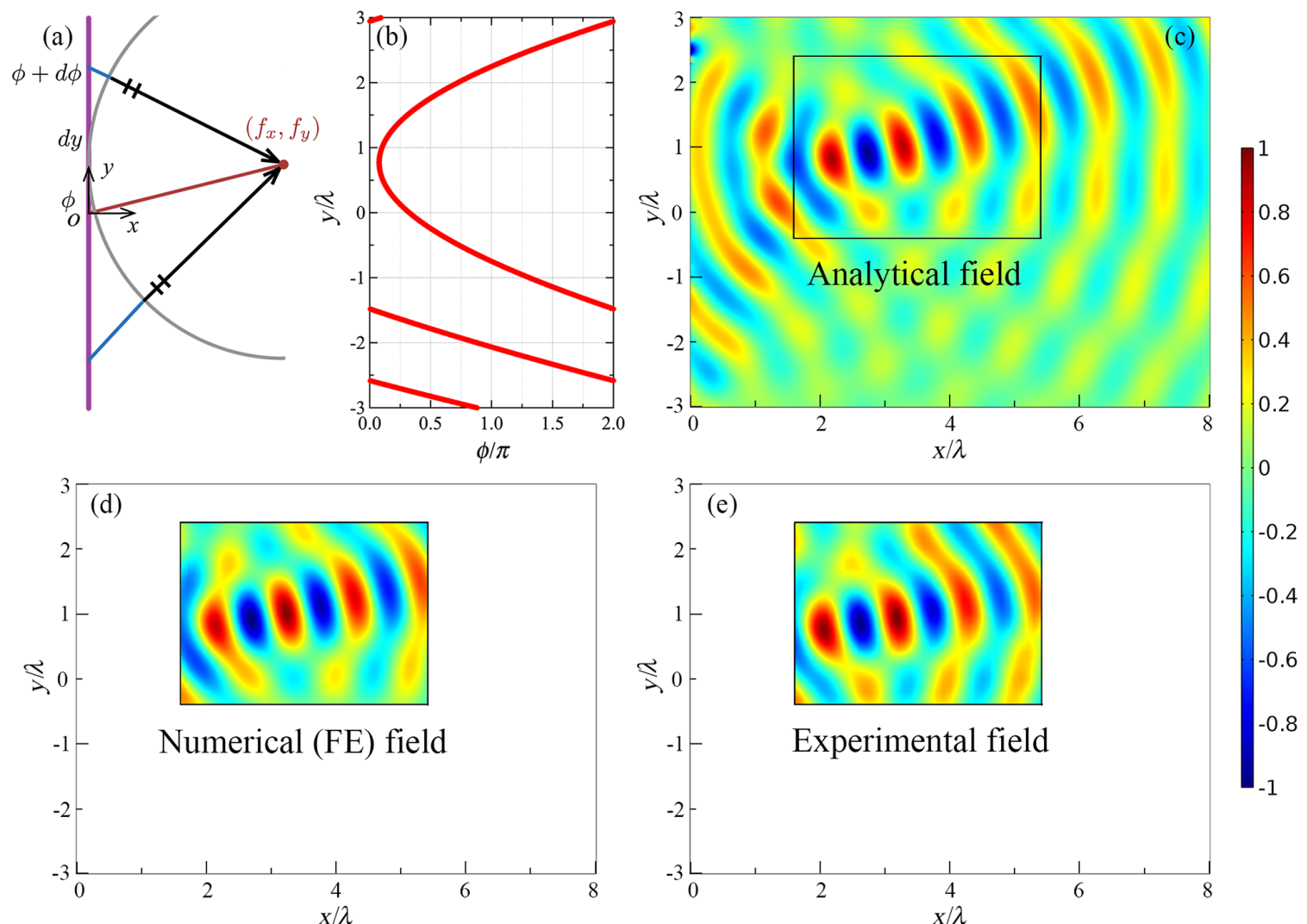

FIG. 4. (a) The schematics for the derivation of the acoustic focusing with focus point located at $\left(f_{x}, f_{y}\right)$. The length of the blue line is the acoustic path distance difference provided by the metasurface. (b) The desired continuous phase profile that needs to be yielded by the metasurfaces with length $2 L=6 \lambda$. (c) The pressure-field $p(x, y)$ distribution predicted by Green's-function theory. The black box indicates the region within which the measurement is performed. (d) The numerical simulation of metasurface composed by labyrinthine units and (e) the experimental results of the pressure-field distribution.

illustrates the design of a metasurface, which is, in fact, a phase mask to convert an incident wave into a nonparaxial beam based on the caustic theory [44]. The relationship between the phase profile $\phi(y)$ and the reflected angle $\theta_{r}$ in the $y$ direction can be expressed as $d \phi(y) / d y=$ $-k_{0} \sin \left(\theta_{r}\right)$. To realize the arbitrary trajectory $y=f(x)$, the phase profile can be expressed as $d \phi(y) / d y=$ $-k_{0}\left\{f^{\prime}(x) / \sqrt{1+\left[f^{\prime}(x)\right]^{2}}\right\}$ with $f^{\prime}(x)=\tan \left(\theta_{r}\right)$ being the slope of the trajectory. For a particular halfcircle trajectory $f(x)=\sqrt{r^{2}-(x-r)^{2}}$ with center at $(x, y)=(r, 0)$, the desired profile can be expressed as $\phi(y)=-k_{0}(y-2 r \sqrt{y / r})$. In the simulations and experiments, the length of the metasurface is $8 \lambda_{0}$ and extends from $y=0$ to $y=8 \lambda_{0}$, and the point source is located at $(x, y)=(1 \mathrm{~m}, 0.4 \mathrm{~m})$. Then, the desired phase profile yielded by metasurface should be $\phi(y)=$ $-k_{0}\left\{(y-0.7 \sqrt{y / 0.35})+\left[\sqrt{(y-0.4)^{2}+1}-1\right]\right\} \quad$ with $r=0.35 \mathrm{~m}$, as shown in Fig. 5(b). The pressure-field distribution analytically predicted by Eq. (3) is shown in Fig. 5(c). The numerical simulations of the metasurface composed by appropriately choosing the labyrinthine unit are shown in Fig. 5(d), and the corresponding experimental results are shown in Fig. 5(e). Because of the fine resolution along the $y$ direction in our simulations and experiments (viz., $\lambda_{0} / 8$ ), good agreement is achieved between the results of the numerical simulation and the experimental measurement. A trivial spatial aliasing effect [46], which accounts for the slight difference between the analytical predictions and the numerical and experimental results, can also be observed due to the inevitable discretization and the quick oscillation of the desired phase profile along the $y$ direction, as shown in Fig. 5(b).

We anticipate that the acoustic metasurface capable of yielding arbitrary acoustic beams will provide remarkable flexibility and open new exciting possibilities in a variety of acoustic-based applications ranging from ultrasound imaging to acoustic device design. For instance, these beams show promise to realize the trapping and guiding of microparticles along arbitrary trajectories, or they may enable circumventing an acoustic obstacle by designing a bypassing caustic. 

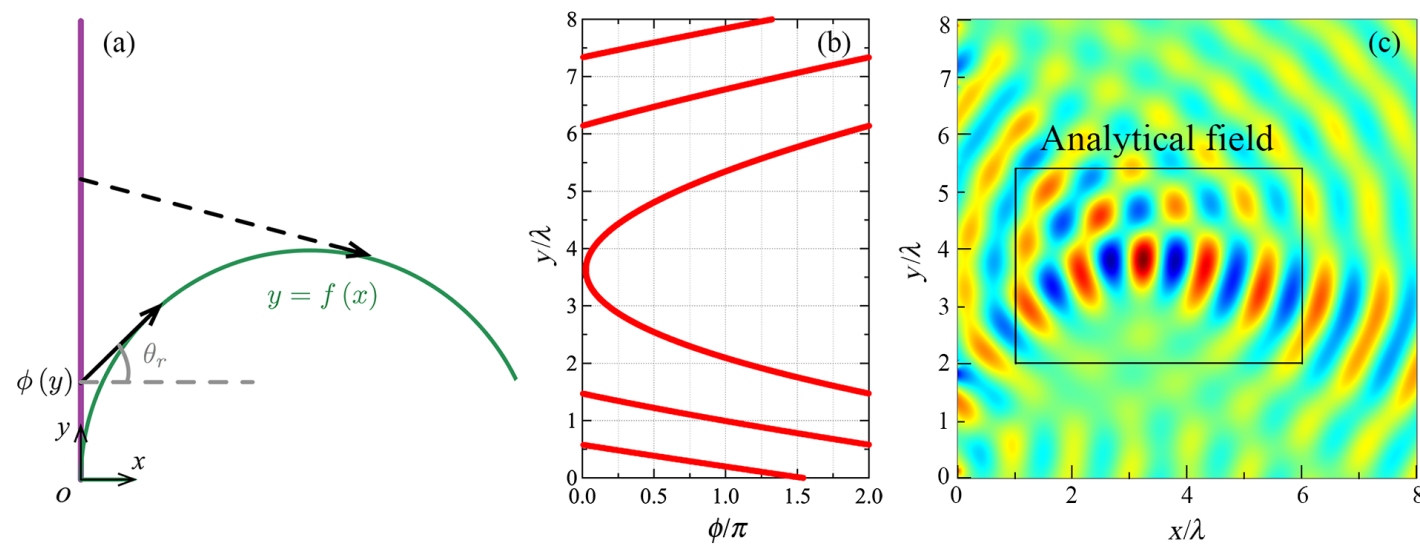

(c)
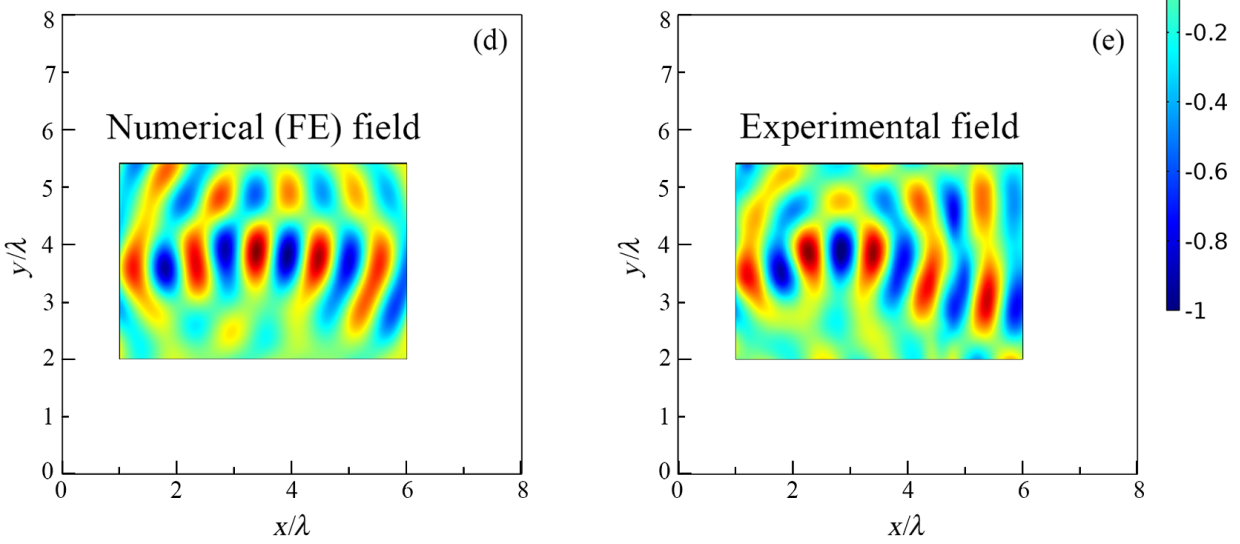

FIG. 5. (a) The schematics for the derivation of the phase profile for the arbitrary trajectory. (b) The desired continuous phase profile that needs to be yielded by the metasurfaces with length $2 L=8 \lambda$. (c) The pressure-field $p(x, y)$ distribution predicted by Green's-function theory. The black box indicates the region within which the measurement is performed. (d) The numerical simulation of the metasurface composed by labyrinthine units and (e) the experimental results of the pressure-field distribution.

\section{CONCLUSION}

In conclusion, we design and experimentally realize acoustic metasurfaces that can fully control reflected waves in the direction and shape of the wave front. A general scheme of designing an acoustic metasurface is developed on the basis of labyrinthine units, which can give an analytical description to the phase shifts. By suitably designing the geometry of the metamaterial units, the metasurfaces with subwavelength thickness can reflect acoustic waves in an unusual yet controllable manner. As particular examples, three kinds of distinct properties of acoustic wave manipulation are demonstrated both theoretically and experimentally, yielding anomalous reflection governed by the generalized Snell's law, mimicking ultrathin planar lenses, and, remarkably, generating nondiffractive beams along an arbitrary trajectory. The results reveal great flexibility in the design of acoustic beams enabled by the free manipulation of a propagating phase with metasurfaces. The realization of acoustic metasurfaces with the capability of controlling acoustic waves arbitrarily should open an avenue to design versatile compact acoustic components and have potential applications in a large variety of fields in which special manipulation of acoustic flux is required, such as ultrasound imaging and caustic engineering.

\section{ACKNOWLEDGMENTS}

We acknowledge helpful discussions with Professor Tao Li. This work is supported by the National Basic Research Program of China (973 Program) (Grants No. 2010CB327803 and No. 2012CB921504), the National Natural Science Foundation of China (Grants No. 11174138, No. 11174139, No. 11222442, No. 81127901, and No. 11274168), Program for New Century Excellent Talents in University (No. NCET-120254), and a project funded by the Priority Academic Program Development of Jiangsu Higher Education Institutions.

Y. L. and X. J. contributed equally to this work.

[1] N. Yu, P. Genevet, M. A. Kats, F. Aieta, J. P. Tetienne, F. Capasso, and Z. Gaburro, Light propagation with phase discontinuities: Generalized laws of reflection and refraction, Science 334, 333 (2011). 
[2] F. Aieta, P. Genevet, M. A. Kats, N. Yu, R. Blanchard, Z. Gaburro, and F. Capasso, Aberration-free ultrathin flat lenses and axicons at telecom wavelengths based on plasmonic metasurfaces, Nano Lett. 12, 4932 (2012).

[3] X. Ni, N. K. Emani, A. V. Kildishev, A. Boltasseva, and V. M. Shalaev, Broadband light bending with plasmonic nanoantennas, Science 335, 427 (2012).

[4] S. Sun, Q. He, S. Xiao, Q. Xu, X. Li, and L. Zhou, Gradientindex meta-surfaces as a bridge linking propagating waves and surface waves, Nat. Mater. 11, 426 (2012).

[5] F. Monticone, N. M. Estakhri, and A. Alu, Full control of nanoscale optical transmission with a composite metascreen, Phys. Rev. Lett. 110, 203903 (2013).

[6] H. Wakatsuchi, S. Kim, J. J. Rushton, and D. F. Sievenpiper, Waveform-dependent absorbing metasurfaces, Phys. Rev. Lett. 111, 245501 (2013).

[7] Y. Mazor and B. Z. Steinberg, Metaweaves: Sector-way nonreciprocal metasurfaces, Phys. Rev. Lett. 112, 153901 (2014).

[8] P. M. Morse and K. U. Ingard, Theoretical Acoustics (Princeton University Press, Princeton, NJ, 1986).

[9] Z. Liu, X. Zhang, Y. Mao, Y. Y. Zhu, Z. Yang, C. T. Chan, and P. Sheng, Locally resonant sonic materials, Science 289, 1734 (2000).

[10] J. Li and C. T. Chan, Double-negative acoustic metamaterial, Phys. Rev. E 70, 055602 (2004).

[11] N. Fang, D. Xi, J. Xu, M. Ambati, W. Srituravanich, C. Sun, and $\mathrm{X}$. Zhang, Ultrasonic metamaterials with negative modulus, Nat. Mater. 5, 452 (2006).

[12] Y. Ding, Z. Liu, C. Qiu, and J. Shi, Metamaterial with simultaneously negative bulk modulus and mass density, Phys. Rev. Lett. 99, 093904 (2007).

[13] Z. Yang, J. Mei, M. Yang, N. H. Chan, and P. Sheng, Membrane-type acoustic metamaterial with negative dynamic mass, Phys. Rev. Lett. 101, 204301 (2008).

[14] S. H. Lee, C. M. Park, Y. M. Seo, Z. G. Wang, and C. K. Kim, Composite acoustic medium with simultaneously negative density and modulus, Phys. Rev. Lett. 104, 054301 (2010).

[15] J. Christensen and F. J. Garcia de Abajo, Anisotropic metamaterials for full control of acoustic waves, Phys. Rev. Lett. 108, 124301 (2012).

[16] Z. Liang and J. Li, Extreme acoustic metamaterial by coiling up space, Phys. Rev. Lett. 108, 114301 (2012).

[17] Y. Xie, B. I. Popa, L. Zigoneanu, and S. A. Cummer, Measurement of a broadband negative index with spacecoiling acoustic metamaterials, Phys. Rev. Lett. 110, 175501 (2013).

[18] M. Yang, G. Ma, Z. Yang, and P. Sheng, Coupled membranes with doubly negative mass density and bulk modulus, Phys. Rev. Lett. 110, 134301 (2013).

[19] Z. Liang, T. Feng, S. Lok, F. Liu, K. B. Ng, C. H. Chan, J. Wang, S. Han, S. Lee, and J. Li, Space-coiling metamaterials with double negativity and conical dispersion, Sci. Rep. 3, 1614 (2013).

[20] J. Li, L. Fok, X. Yin, G. Bartal, and X. Zhang, Experimental demonstration of an acoustic magnifying hyperlens, Nat. Mater. 8, 931 (2009).

[21] S. Zhang, L. Yin, and N. Fang, Focusing ultrasound with an acoustic metamaterial network, Phys. Rev. Lett. 102, 194301 (2009).
[22] J. Zhu, J. Christensen, J. Jung, L. Martin-Moreno, X. Yin, L. Fok, X. Zhang, and F. J. Garcia-Vidal, A holey-structured metamaterial for acoustic deep-subwavelength imaging, Nat. Phys. 7, 52 (2011).

[23] B. Liang, B. Yuan, and J.C. Cheng, Acoustic diode: Rectification of acoustic energy flux in one-dimensional systems, Phys. Rev. Lett. 103, 104301 (2009).

[24] B. Liang, X. S. Guo, J. Tu, D. Zhang, and J. C. Cheng, An acoustic rectifier, Nat. Mater. 9, 989 (2010).

[25] X. F. Li, X. Ni, L. A. Feng, M. H. Lu, C. He, and Y. F. Chen, Tunable unidirectional sound propagation through a soniccrystal-based acoustic diode, Phys. Rev. Lett. 106, 084301 (2011).

[26] Y. Li, B. Liang, Z. M. Gu, X. Y. Zou, and J. C. Cheng, Unidirectional acoustic transmission through a prism with near-zero refractive index, Appl. Phys. Lett. 103, 053505 (2013).

[27] R. Fleury, D. L. Sounas, C. F. Sieck, M. R. Haberman, and A. Alu, Sound isolation and giant linear nonreciprocity in a compact acoustic circulator, Science 343, 516 (2014).

[28] B. I. Popa, L. Zigoneanu, and S. A. Cummer, Experimental acoustic ground cloak in air, Phys. Rev. Lett. 106, 253901 (2011).

[29] S. Zhang, C. Xia, and N. Fang, Broadband acoustic cloak for ultrasound waves, Phys. Rev. Lett. 106, 024301 (2011).

[30] L. Zigoneanu, B. I. Popa, and S. A. Cummer, Threedimensional broadband omnidirectional acoustic ground cloak, Nat. Mater. 13, 352 (2014).

[31] J. Mei, G. Ma, M. Yang, Z. Yang, W. Wen, and P. Sheng, Dark acoustic metamaterials as super absorbers for lowfrequency sound, Nat. Commun. 3, 756 (2012).

[32] G. Ma, M. Yang, S. Xiao, Z. Yang, and P. Sheng, Acoustic metasurface with hybrid resonances, Nat. Mater. 13, 873 (2014).

[33] Y. Li, B. Liang, Z. M. Gu, X. Y. Zou, and J. C. Cheng, Reflected wavefront manipulation based on ultrathin planar acoustic metasurfaces, Sci. Rep. 3, 2546 (2013).

[34] J. Zhao, B. Li, Z. Chen, and C. W. Qiu, Manipulating acoustic wavefront by inhomogeneous impedance and steerable extraordinary reflection, Sci. Rep. 3, 2537 (2013).

[35] Y. Xie, A. Konneker, B.-I. Popa, and S. A. Cummer, Tapered labyrinthine acoustic metamaterials for broadband impedance matching, Appl. Phys. Lett. 103, 201906 (2013).

[36] F. Cervera, L. Sanchis, J. V. Sanchez-Perez, R. MartinezSala, C. Rubio, F. Meseguer, C. Lopez, D. Caballero, and J. Sanchez-Dehesa, Refractive acoustic devices for airborne sound, Phys. Rev. Lett. 88, 023902 (2001).

[37] Y. Li, B. Liang, X. Tao, X. F. Zhu, X. Y. Zou, and J. C. Cheng, Acoustic focusing by coiling up space, Appl. Phys. Lett. 101, 233508 (2012).

[38] Y. Li, B. Liang, X. Y. Zou, and J. C. Cheng, Extraordinary acoustic transmission through ultrathin acoustic metamaterials by coiling up space, Appl. Phys. Lett. 103, 063509 (2013).

[39] T. Frenzel, J. D. Brehm, T. Buckmann, R. Schittny, M. Kadic, and M. Wegener, Three-dimensional labyrinthine acoustic metamaterials, Appl. Phys. Lett. 103, 061907 (2013). 
[40] Y. Li, G. K. Yu, B. Liang, X. Y. Zou, G. Y. Li, S. Cheng, and J. C. Cheng, Three-dimensional ultrathin planar lenses by acoustic metamaterials, Sci. Rep. 4, 6830 (2014).

[41] G. A. Siviloglou and D. N. Christodoulides, Accelerating finite energy airy beams, Opt. Lett. 32, 979 (2007).

[42] L. Li, T. Li, S. M. Wang, C. Zhang, and S. N. Zhu, Plasmonic airy beam generated by in-plane diffraction, Phys. Rev. Lett. 107, 126804 (2011).

[43] P. Zhang, Y. Hu, T. Li, D. Cannan, X. Yin, R. Morandotti, Z. Chen, and X. Zhang, Nonparaxial mathieu and Weber accelerating beams, Phys. Rev. Lett. 109, 193901 (2012).
[44] E. Greenfield, M. Segev, W. Walasik, and O. Raz, Accelerating light beams along arbitrary convex trajectories, Phys. Rev. Lett. 106, 213902 (2011).

[45] P. Zhang, T. Li, J. Zhu, X. Zhu, S. Yang, Y. Wang, X. Yin, and $X$. Zhang, Generation of acoustic self-bending and bottle beams by phase engineering, Nat. Commun. 5, 4316 (2014).

[46] D. H. Johnson and D. E. Dudgeon, Array Signal Processing: Concepts and Techniques (Prentice-Hall, Englewood Cliffs, NJ, 1993). 